\title{
2 Aim of the thesis
}

The aim oft this thesis was to identify novel monogenic disorders leading to primary immunodeficiencies using state of the art technologies such as exome sequencing and homozygosity mapping.

The identification and functional characterization of the underlying genetic defects will help to understand the pathogenesis of these diseases and may open novel therapeutic opportunities for targeted therapy of affected individuals. Moreover, the identification of keymolecules in the development of autoimmunity and/or lymphoproliferation will help to understand the pathophysiology and pathogenesis not only of these monogenic diseases, but can be extrapolated to a large group of diseases involving autoimmunity. 